Pacific Journal of Mathematics

INVERSE SYSTEMS OF GROUP-VALUED MEASURES 


\section{INVERSE SYSTEMS OF GROUP-VALUED MEASURES}

\section{Hugh Millington and Maurice Sion}

In this paper a basic theory is developed for inverse (or projective) systems of group-valued measures. This theory parallels the one for nonnegative measures. However, many of the results are new even in the real case.

The main tools for dealing with group-valued measures are the concepts and results given by Sion in "Outer measures with values in a topological group", Proc. London Math. Soc., 19 (1969), 89-106. When dealing with inverse systems the point of view adopted is that of Mallory and Sion, "Limits of inverse systems of measures". Ann. Inst. Fourier, Tome 21, Fasc. 1 (1971) 25-57. This viewpoint involves finding a limit measure first on a large space $\Lambda$ and then studying conditions under which this will yield a limit measure on some subset of $\Lambda$. By introducing the concept of almost-sequential maximality, this paper not only extends known results but is also able to indicate a connection between "abstract" and "topological" methods for producing a limit measure.

In the last section the results obtained are applied to cylinder measures. Here again the viewpoint adopted differs somewhat from the usual one, even for nonnegative measures, and enables one to study a variety of possibilities for a target space on which to place a limit measure.

o. Notation and basic notions. Throughout this paper, $\omega$ is the set of nonnegative integers, $\boldsymbol{R}$ is the real line, $\Gamma$ is a commutative, complete, Hausdorff, topological group with identity $o$ under the operations + and - .

For any sets $A$ and $B$,

$$
A \sim B=\{x: x \in A \text { and } x \notin B\} .
$$

For any subsets $A$ and $B$ of $\Gamma$ and $n \in w$,

$$
\begin{aligned}
A+B & =\{x+y: x \in A \text { and } y \in B\}, \\
n A & =A+\cdots+A \quad(n \text { terms }) .
\end{aligned}
$$

Definition 0.1. For any family $\mathscr{C}$ of sets, $\mathscr{H}$ is $\omega$-compact iff every countable subfamily of $\mathscr{H}$ with the finite intersection property has a nonempty intersection.

Definitions 0.2. For any function $\xi$ on the family of all subsets of some space $\Omega$ to $\Gamma$, 
(1) $A$ is $\xi$-measurable iff $A \subset \Omega$ and, for every

$$
T \subset \Omega, \xi(T)=\xi(T \cap A)+(T \sim A) .
$$

(2) $M_{\xi}=\{A: A$ is $\xi$-measurable $\}$.

(3) $A$ is $\xi$-null iff $A \subset \Omega$ and, for every $\alpha \subset A, \xi(\alpha)=o$.

(4) $\xi$ is an outer measure on $\Omega$ iff.

(i ) $M_{\xi}$ is a $\sigma$-field and $\xi$ is $\sigma$-additive on $M_{\xi}$,

(ii) for any $A \subset \Omega, \xi(A)=\operatorname{limit} \xi(\alpha)$ as $\alpha$ runs over $\left\{\alpha \in M_{\xi}: A \subset \alpha\right\}$ directed by $\supset$.

Definition 0.3. For any function $\tau$ on a family $\mathscr{A}$ of sets to $\Gamma$ and $\mathscr{H} \subset \mathscr{A}, \mathscr{H}$ is an inner family for $\tau$ iff, for every $A \in \mathscr{A}$ and neighborhood $U$ of $\tau(A)$, there exists $H \in \mathscr{C}$ such that $H \subset A$ and, for every $\alpha \in \mathscr{A}$,

$$
H \subset \alpha \subset A \Longrightarrow \tau(\alpha) \in U .
$$

Definition 0.4. For any topological space $\Omega, \xi$ is a Radón outer measures on $\Omega$ iff $\xi$ is an outer measure on $\Omega$ such that

(i) closed sets are $\xi$-measurable,

(ii) the closed, compact sets form an inner family for $\left(\xi / M_{\xi}\right)$.

In the sequel, we shall need the following theorems which generalize well-known results for real-valued measures.

THeORem 0.5. Let $\mathscr{A}$ be a field of subsets of a space $\Omega$ and $\tau$ be a $\sigma$-additive function on $\mathscr{A}$ to $\Gamma$ such that, for any monotone sequence ${ }^{(1)} \alpha$ in $\mathscr{A}, \lim _{n} \tau\left(\alpha_{n}\right) \in \Gamma$. If

$$
\tau^{\prime}\left(\cup \alpha_{n}\right)=\lim _{n} \tau\left(\alpha_{n}\right) \quad \text { for } \alpha_{n} \subset \alpha_{n+1} \in \mathscr{A}
$$

and, for any $A \subset \Omega, \tau^{*}(A)=\operatorname{limit} \tau^{\prime}(\beta)$ as $\beta$ runs over

$$
\left\{\beta \in \mathscr{A}_{0}: A \subset \beta\right\}
$$

directed by $\supset$

then $\tau^{*}$ is an outer measure on $\Omega$ such that $\mathscr{A} \subset M_{\tau^{*}}$ and $\tau^{*} / \mathscr{A}=\tau$.

Proof. See Sion [11] Theorem 3.3.

THEOREM 0.6. Let $\Omega$ be a regular topological space, $\mathscr{K}$ be the family of closed, compact subsets of $\Omega$, and $\tau$ be a $\sigma$-additive function on $\mathscr{K}$ to $\Gamma$ such that

(i) for any ascending sequence $C$ in $\mathscr{K}, \lim _{n}\left(C_{n}\right) \in \Gamma$,

(ii) for any neighborhood $U$ of $o$ and $C \in \mathscr{K}$, there exists an open $G$ such that $C \subset G$ and, for every $C^{\prime} \in \mathscr{K}$,

1 Depending on context, letters ( $\alpha, E$, etc.) may denote a single set or a sequence $\left(\left\{\alpha_{n}\right\},\left\{E_{n}\right\}\right)$ of sets. 


$$
C \subset C^{\prime} \subset G \Longrightarrow \tau(C)-\tau\left(C^{\prime}\right) \in U .
$$

If, for any open $G, \rho^{\prime}(G)=\operatorname{limit} \tau(C)$ as $C$ runs over

$$
\{C \in \mathscr{K}: C \subset G\} \text { directed by } \subset \text { and, for any } A \subset \Omega \text {, }
$$

$\rho(A)=\operatorname{limit} \rho^{\prime}(G)$ as $G$ runs over

$\{G: G$ is open and $A \subset G\} \quad$ directed by $\supset$,

then $\rho$ is a Radón outer measure on $\Omega$ and $(\rho / \mathscr{K})=\tau$.

Proof. See Sion [11] Theorem 6.3.

Lemma 0.7. For any outer measure $\xi, A \in M_{\xi}$ iff, for every neighborhood $U$ of $o$, there exist $A^{\prime}, A^{\prime \prime} \in M_{\xi}$ such that $A^{\prime} \subset A \subset A^{\prime \prime}$ and $\left(\alpha \subset A^{\prime \prime} \sim A^{\prime} \Rightarrow \xi(\alpha) \in U\right)$.

1. Inverse systems of abstract measures. Throughout this paper, $\mathscr{F}$ is an index set directed by a relation $<$;

$(S, r)$ is an inverse system of spaces indexed by $(\mathscr{F},<)$, i.e.,

$S_{E}$ is an abstract space for $E \in \mathscr{F}$,

$r_{E, F}: S_{F} \rightarrow S_{E}$ is surjective for $E<F$,

$r_{E, E}$ is the identity map,

$r_{E G}=r_{E F} \circ r_{F G} \quad$ for $E<F<G$;

$(\Lambda, p)$ is a limit of $(S, r)$, i.e.,

$\Lambda$ is an abstract space,

$p_{E}: \Lambda \rightarrow S_{E}$ is surjective for $E \in \mathscr{F}$,

$p_{E}=r_{E F} \circ p_{F} \quad$ for $E<F$;

$\mu$ is a system of outer measures on $S$, i.e., for each $E \in \mathscr{F}$, $\mu_{E}$ is a $\Gamma$-valued outer measure on $S_{E}$ and $M_{E}=M_{\mu_{E}}$.

DEFINITIONS 1.1. (1) $\mu$ is an inverse system of measures over $(S, r)$ iff, for $E, F \in \mathscr{F}$ with $E<F$ and $A \in M_{E}$,

$$
r_{E F}^{-1}[A] \in M_{F} \quad \text { and } \quad \mu_{F}\left(r_{E F}^{-1}[A]\right)=\mu_{E}(A) .
$$

(2) $\mu$ is monotone iff, for any ascending sequence $E$ in $\mathscr{F}$ and any sequence $A$ with

$$
A_{n} \in M_{E_{n}} \quad \text { and } \quad r_{E_{n} E_{n+1}^{-1}}\left[A_{n}\right] \subset A_{n+1} \text { for } n \in \omega,
$$

we have $\lim _{n} \mu_{E_{n}}(A) \in \Gamma$.

Note: Any inverse system of nonnegative measures is monotone.

DeFinition 1.2. For any $Q \subset \Lambda$,

(1) $\xi$ is a limit of $\mu$ on $\Omega$ iff $\xi$ is a $\Gamma$-valued outer measure on 
$\Omega$ such that, for every $E \in \mathscr{F}$ and $A \in M_{E}$,

$$
\left(\Omega \cap p_{E}^{-1}[A]\right) \in M_{\xi} \quad \text { and } \quad \xi\left(\Omega \cap p_{E}^{-1}[A]\right)=\mu_{E}(A) .
$$

(2) $\operatorname{Cyl} \Omega=\left\{\Omega \cap p_{E}^{-1}[A] ; E \in \mathscr{F}\right.$ and $\left.A \in M_{E}\right\}$.

(3) $\tau_{\Omega}$ is the function $\tau$ on $\operatorname{Cyl} \Omega$ such that

$$
\tau\left(\Omega \cap p_{E}^{-1}[A]\right)=\mu_{E}(A) \quad \text { for } E \in \mathscr{F} \text { and } A \in M_{E} .
$$

(4) $\tau_{\Omega}^{*}$ is the outer measure on $\Omega$ generated by $\tau_{\Omega}$ as in Theorem 0.5 .

Remarks. (1) Cyl $\Omega$ is a field of subsets of $\Omega$.

(2) $\tau_{\Omega}$ is well defined iff, for every $E \in \mathscr{F}$ and $A \in M_{E}$,

$$
\Omega \cap p_{E}^{-1}[A]=\phi \Longrightarrow \mu_{E}(A)=0 .
$$

(This will clearly be the case if $p_{E}[\Omega]=S_{E}$ for $E \in \mathscr{F}$ ).

(3) When $\tau_{Q}$ is well defined, it is finitely additive. Moreover, if $\mu$ is a monotone inverse system of measures then, for any monotone sequence $\alpha$ in $\operatorname{Cyl} \Omega, \lim _{n} \tau_{\Omega}\left(\alpha_{n}\right) \in \Gamma$. For such a $\mu$ therefore, by Theorem 0.5 , we see that $\tau_{\Omega}^{*}$ is well defined iff $\tau_{\Omega}$ is $\sigma$-additive, in which case $\tau_{\Omega}^{*}$ is an outer measure on $\Omega$ which extends $\tau_{\Omega}$.

Assumption 1.3. For the remainder of this section, we suppose $\mu$ is a monotone inverse system of measures over $(S, r)$.

Remark (3) above then yields immediately the basic result.

Lemma 1.4. For any $\Omega \subset \Lambda$, there exists a limit of $\mu$ on $\Omega$ iff $\tau_{\Omega}$ is $\sigma$-additive, in which case $\tau_{\Omega}^{*}$ is such a limit.

In view of the above lemma, all the known theorems about the existence of a limit of $\mu$ on $\Lambda$ when $\Gamma=R$ can be extended to the general situation discussed here with little or no difficulty. For the remainder of this section, we consider a problem which has received little attention in the literature, except in special cases, namely that of finding conditions under which the existence of a limit of $\mu$ on $\Lambda$ implies the existence of a limit of $\mu$ on a subset of $\Lambda$.

We first note the following key lemma.

Lemma 1.5. Suppose $\tau_{A}$ is $\sigma$-additive, $\lambda=\tau_{A}^{*}$ and $\Omega \subset \Lambda$. Then $\tau_{\Omega}$ is $\sigma$-additive iff $\lambda(\Omega \cap B)=\lambda(B)$ for $B \in \operatorname{Cyl} \Lambda$.

Proof. (1) Suppose $\tau_{\Omega}$ is $\sigma$-additive and $B \in \operatorname{Cyl} \Lambda$.

For any ascending sequence $\beta$ in $\operatorname{Cyl} \Lambda$ with

$$
\Omega \cap B \subset \bigcup_{n \in \omega} \beta_{n} \subset B
$$


since $Q \cap \beta_{n} \in \operatorname{Cyl} \Omega$, we have

$$
\lim _{n} \tau_{\Lambda}\left(\beta_{n}\right)=\lim _{n} \tau_{\Omega}\left(\Omega \cap \beta_{n}\right)=\tau_{\Omega}(\Omega \cap B)=\tau_{\Lambda}(B)=\lambda(B) .
$$

We conclude therefore $\lambda(\Omega \cap B)=\lambda(B)$.

(2) If $\lambda(\Omega \cap B)=\lambda(B)$ for $B \in \operatorname{Cyl} \Lambda$ then the restriction of $\lambda$ to the subsets of $\Omega$ is clearly a limit of $\mu$ on $\Omega$ and hence $\tau_{\Omega}$ is $\sigma$ additive.

We then have the following.

THEorem 1.6. Suppose $\tau_{\Lambda}$ is $\sigma$-additive, $\lambda=\tau_{\Lambda}^{*}$ and $\Omega \subset \Lambda . \quad$ If, for any ascending sequence $E$ in $\mathscr{F}$, the set

$N(E)$

$=\left\{f \in \Lambda\right.$ : There does not exist $g \in \Omega$ with $p_{E_{n}}(g)=p_{E_{n}}(f)$ for all $\left.n \in \omega\right\}$ is $\lambda$-null then $\tau_{\Omega}$ is $\sigma$-additive.

Proof. We shall show that the hypothesis implies that any sequence $\beta$ in $\operatorname{Cyl} \Lambda$ which covers $\Omega$ must cover almost all of $\Lambda$. Indeed, let $\beta_{n}=p_{E_{n}}^{-1}\left[A_{n}\right]$ with $E_{n}<E_{n+1}$ and $A_{n} \in M_{E_{n}}$ for $n \in \omega$ and $\Omega \subset \mathbf{U}_{n \in \omega} \beta_{n}$.

Then we must have

$$
\Lambda \sim \bigcup_{n \in \omega} \beta_{n} \subset N(E)
$$

for, if $f \in \Lambda$ and there exists $g \in \Omega$ with $p_{E_{n}}(g)=p_{E_{n}}(f)$ for all $n \in \omega$ then, since $g \in \beta_{m}$ for some $m \in \omega$, we have

$$
p_{E_{m}}(f)=p_{E_{m}}(g) \in A_{m} \text {, so } f \in p_{E_{m}}^{-1}\left[A_{m}\right]=\beta_{m} \text {. }
$$

Since $N(E)$ is $\lambda$-null, we conclude

$$
\lambda\left(\bigcup_{n \in \omega} \beta_{n}\right)=\lambda(\Lambda)
$$

and therefore, for any $B \in \operatorname{Cyl} \Lambda, \lambda(\Omega \cap B)=\lambda(B)$. Application of Lemma 1.5. then yields the desired conclusion.

We now state conditions on $\Omega$ in terms of the system $\mu$, rather than in terms of the limit $\lambda$, which guarantee the existence of a limit of $\mu$ on $\Omega$.

Definition 1.7. For any neighborhood $U$ of $o, E \in \mathscr{F}$ and $A \subset S_{E}, A$ is $U$-small iff, for every $F \in \mathscr{F}$ with $E<F$,

$$
\alpha \subset r_{E F}^{-1}[A] \Longrightarrow \mu_{F}(\alpha) \in U .
$$

DEFinitions 1.8. For any $\Omega \subset \Lambda$, 
(1) $\Omega$ is sequentially maximal iff, for every ascending sequence $E$ in $\mathscr{F}$ and sequence $f$ with

$$
f_{n} \in S_{E_{n}} \quad \text { and } \quad r_{E_{n} E_{n+1}}\left(f_{n+1}\right)=f_{n} \quad \text { for } n \in \omega,
$$

thers exists a $g \in \Omega$ with $p_{E_{n}}(g)=f_{n}$ for all $n \in \omega$.

(2) $\Omega$ is almost sequentially maximal iff, for every neighborhood $U$ of $o$ and ascending sequence $E$ in $\mathscr{F}$, there exists a sequence $A$ such that

(i) $A_{n} \in M_{E_{n}}, r_{E_{n} E_{n+1}}^{-1}\left[A_{n}\right] \subset A_{n+1}$ and $A_{n}$ is $U$-small for $n \in \omega$,

(ii) for any sequence $f$ with

$$
f_{n} \in S_{E_{n}} \sim A_{n} \quad \text { and } \quad r_{E_{n} E_{n+1}}\left(f_{n+1}\right)=f_{n} \quad \text { for } n \in \omega,
$$

there exists a $g \in \Omega$ with $p_{E_{n}}(g)=f_{n}$ for all $n \in \omega$,

Definitions 1.9. For any family $\mathscr{C}$ of subsets of $\Lambda$,

(1) $\mu$ is $\mathscr{C}$-tight iff, for every neighborhood $U$ of $o$, there exists $C \in \mathscr{C}$ such that $\left(S_{E} \sim p_{E}[C]\right)$ is $U$-small for every $E \in \mathscr{F}^{(2)}$

(2) $\mu$ is sequentially $\mathscr{C}$-tight iff, for every nighborhood $U$ of $o$ and ascending sequence $E$ in $f$, there exists $C \in \mathscr{C}$ such that $\left(S_{E_{n}} \sim\right.$ $\left.p_{E_{n}}[C]\right)$ is $U$-small for every $n \in \omega$.

REMARK. The condition of sequential maximality is extensively used in the literature in connection with finding a limit of $\mu$, especially when dealing with abstract measures. (see, e.g. 2, 3, 4, 5).

However, as the observation at the end of $\S 3$ shows, such a condition is much too strong to be useful in many applications. Hence, when dealing with topological measures, one finds the condition of tightness frequently used (see, e.g. $7,8,9,10$ ).

No relation seems to exist between the two approaches. By using the weaker concepts of almost sequential maximality and of sequential tightness, introduced above, we show the connection between the two notions while extending known results even when $\Gamma=\boldsymbol{R}$.

THeOREM 1.10. If $\mu$ has a limit on $\Lambda$ and $\Omega \subset \Lambda$ is almost sequentially maximal then $\mu$ has a limit on $\Omega$.

Proof. We shall show that the hypothesis of Theorem 1.6 is satisfied. Let $\lambda=\tau_{A}^{*}, E$ be an ascending sequence in $\mathscr{F}$ and $\beta \subset\left\{f \in \Lambda\right.$ : there does not exist $g \in \Omega$ with $p_{E_{n}}(g)=p_{E_{n}}(f)$ for all $\left.n \in \omega\right\}$.

To see that $\lambda(\beta)=o$, given any neighborhood $U$ of $o$, let $A$ be

2 See Ch. I of the forthcoming book by Laurent Schwartz "Radón measures on topological spaces." Tata Institute, Bombay. 
a sequence satisfying Conditions (i) and (ii) of Definition 1.8.2. Then

$$
\beta \subset \bigcup_{n \in \omega} p_{E_{n}}^{-1}\left[A_{n}\right]
$$

for any $k \in \omega$,

$$
\bigcup_{n=0}^{k} p_{E_{k}}^{-1}\left[A_{n}\right]=p_{E_{k}}^{-1}\left[A_{k}\right]
$$

and for any $\alpha \in \operatorname{Cyl} \Lambda$,

$$
\alpha \subset p_{E_{k}}^{-1}\left[A_{k}\right] \Longrightarrow \tau_{A}(\alpha) \in U .
$$

Hence $\lambda(\beta) \in$ closure $U$. Since $U$ is arbitrary, we conclude $\lambda(\beta)=0$.

THEOREM 1.11. Suppose $\Omega \subset \Lambda$ and $\mathscr{C}$ is an $\omega$-compact family of subsets of $\Omega$ such that, for $C \in \mathscr{C}, E \in \mathscr{F}$ and $f \in S_{E}, p_{E}[C] \in M_{E}$ and $\left(C \cap p_{E}^{-1}[f]\right) \in \mathscr{C}$. If $\mu$ is sequentially $\mathscr{C}$-tight then $\Omega$ is almost sequentially maximal.

Proof. Given a neighborhood $U$ of $o$ and an ascending sequence $E$ in $\mathscr{F}$, choose $C \in \mathscr{C}$ so that

$$
S_{E_{n}} \sim p_{E_{n}}[C] \text { is } U \text {-small for } n \in \omega,
$$

and let

$$
A_{n}=S_{E_{n}} \sim p_{E_{n}}[C]
$$

Since

$$
p_{E_{n}}[C]=r_{E_{n} E_{n+1}}\left[p_{E_{n+1}}[C]\right]
$$

we have

$$
r_{E_{n} E_{n+1}}^{-1}\left[A_{n}\right] \subset A_{n+1}
$$

Given any sequence $f$ with

$$
f_{n} \in S_{E_{n}} \sim A_{n} \quad \text { and } \quad r_{E_{n} E_{n+1}}\left(f_{n+1}\right)=f_{n},
$$

we see that, for any $k \in \omega$,

$$
\phi \neq C \cap p_{E_{k}}^{-1}\left[f_{k}\right] \subset C \cap \bigcap_{n=0}^{k} p_{E_{n}}^{-1}\left[f_{n}\right] .
$$

Therefore

$$
\bigcap_{n \in \omega}\left(C \cap p_{E_{n}}^{-1}\left[f_{n}\right]\right) \neq \phi
$$

i.e., there exists $g \in C \subset \Omega$ with $p_{E_{n}}(g)=f_{n}$ for all $n \in \omega$.

CoRollary 1.12. Suppose $\mu$ has a limit on $\Lambda, \Omega \subset \Lambda$ and $\mathscr{C}$ is 
an $\omega$-compact family of subsets of $\Omega$ such that, for $C \in \mathscr{C}, E \in \mathscr{F}$ and $f \in S_{E}, p_{E}[C] \in M_{E}$ and $C \cap p_{E}^{-1}[f] \in \mathscr{C}$. If $\mu$ is sequentially $\mathscr{C}$ tight then $\mu$ has a limit on $\Omega$.

2. Radón systems. We suppose now that, for each $E \in \mathscr{F}, S_{E}$ is a topological space and $\mathscr{K}_{E}$ is the family of closed, compact subsets of $S_{E}$.

DeFinition 2.1. $\mu$ is a Radón system iff, $\mu$ is a monotone, inverse system of measures such that, for each $E \in \mathscr{F}$,

(i) closed subsets of $S_{E}$ are $\mu_{E}$-measurable and

(ii) for every $A \in M_{E}$ and neighborhood $U$ of $o$, there exists $C \in \mathscr{K}_{E}$ such that $C \subset A$ and $(A \sim C)$ is $U$-small.

For Radón systems, we have the following fundamental result.

THEOREM 2.2. Let $\mu$ be a Radon system and $r_{E F}$ be continuous for $E<F$. If $\Lambda$ is almost sequentially maximal then $\tau_{\Lambda}$ is $\sigma$-additive.

Proof. Let $\beta$ be a descending sequence in Cyl $\Lambda$ with

$$
\lim _{n} \tau_{\Lambda}\left(\beta_{n}\right) \neq 0 \text {. }
$$

We shall show that $\bigcap_{n \in \omega} \beta_{n} \neq \phi$. Choose a neighborhood $U$ of $o$ and $N \in \omega$ so that $\tau_{A}\left(\beta_{n}\right) \notin 3 U$ for $n>N$ and let

$$
\beta_{N+n}=p_{E_{n}}^{-1}\left[B_{n}\right] \text { with } E_{n}<E_{n+1} \text { and } B_{n} \in M_{E_{n}} \text {. }
$$

Next, choose a sequence $A$ satisfying Conditions (i) and (ii) of Definition 1.8.2 of almost sequential maximality. Thus,

$$
\mu_{E_{n}}\left(B_{n} \sim A_{n}\right) \notin 2 U \text { and }\left(B_{n+1} \sim A_{n+1}\right) \subset r_{E_{n} E_{n}+1}^{-1}\left[B_{n} \sim A_{n}\right] .
$$

Let $V$ be a sequence of neighborhoods of $o$ with

$$
\sum_{i=0}^{n} V_{i} \subset U
$$

and, by recursion, choose $C_{n} \in \mathscr{K}_{E_{n}}$ so that $C_{n} \subset B_{n} \sim A_{n}$,

$$
\begin{gathered}
C_{n+1} \subset r_{E_{n} E_{n+1}}^{-1}\left[C_{n}\right], \\
\mu_{E_{n}}\left(C_{n}\right)+\sum_{i=0}^{n} V_{i} \not \subset U \text { so } C_{n} \neq \varnothing .
\end{gathered}
$$

Since $\left\{p_{E_{n}}^{-1}\left[C_{n}\right] ; n \in \omega\right\}$ forms a filter base, let $\mathscr{H}$ be the ultra filter induced by it. Then $p_{E_{n}}[\mathscr{H}]$ is an ultrafilter in $C_{n}$ and hence there exits $f_{n} \in C_{n}$ with $f_{n}=$ limit $p_{E_{n}}[\mathscr{C}]$. Since $r_{E_{n} E_{n+1}}$ is continuous, we 
have

$$
r_{E_{n} E_{n+1}}\left(f_{n+1}\right)=f_{n}
$$

hence there exists $g \in \Lambda$ such that $p_{E_{n}}(g)=f_{n}$ for $n \in \omega$, i.e.,

$$
g \in \bigcap_{n \in \omega} p_{E_{n}}^{-1}\left[C_{n}\right] \subset \bigcap_{n \in \omega} \beta_{n} .
$$

Remarks. Even when $\Gamma=\boldsymbol{R}$, the above theorem extends known results in that it uses the weaker hypothesis of almost sequential maximality instead of sequential maximality. As we shall see, this is crucial in connecting "topological" and "abstract" methods for getting limits. Variations of it involving weaker conditions on the $r_{E F}$ and replacing the $K_{E}$ by more general families as in [3], [4], can also be obtained by using slightly different arguments than those used in the above proof.

We now turn our attention to some $\Omega \subset \Lambda$ on which a topology is given and try to determine when $\mu$ has a Radon limit measure on $\Omega$.

Assnmptions 2.3. For the remainder of this section, we assume $S_{E}$ is a Hausdorff, regular, topological space for $E \in \mathscr{F}$; $r_{E F}$ is continuous for $E<F$;

$$
f, g \in \Lambda \text { and } f \neq g \Longrightarrow p_{E}(f) \neq p_{E}(g) \text { for some } E \in \mathscr{F} \text {; }
$$

$\Omega \subset \Lambda, \Omega$ is a regular topological space and $p_{E} / \Omega$ is continuous for $E \in f ; \mu$ is a Radon system.

Thus, $\Omega$ is a Hausdorff space and we let $\mathscr{K}_{\alpha}$ be the family of compact subsets of $\Omega$.

Our main tool for constructing a Radon measure on $\Omega$ is Theorem 0.6. The following lemma enables us to check that the hypotheses of the theorem are satisfied.

Lemma 2.4. Suppose $\tau_{A}$ is $\sigma$-additive and $\lambda=\tau_{1}^{*}$. Then

(1) $\lambda$ is $\sigma$-additive on $\mathscr{K}_{s}$.

(2) for any $C \in \mathscr{K}_{2}$ and neighborhood $U$ of $o$, there exists an open $G \in \operatorname{Cyl} \Omega$ such that $C \subset G$ and

$$
C \subset A \subset G \Longrightarrow \lambda(C)-\lambda(A) \in U,
$$

(3) for any $C \in \mathscr{K}_{2}$,

$$
\lambda(C)=\text { limit } \mu_{F}\left(p_{F}[C]\right) \text { as } F \text { runs over } \mathscr{F} .
$$

Proof. Let $\mathscr{C}$ denote the family of subsets of $\Lambda$ which are compact in the weakest topology on $\Lambda$ under which $p_{E}$ is continuous 
for all $E \in \mathscr{F}$. Then $\mathscr{K}_{\Omega} \subset \mathscr{C}$.

(1) We first check that $\lambda$ is finitely additive on $\mathscr{C}$. Indeed, given disjoint $C, C^{\prime} \in \mathscr{C}$, since the open elements in Cyl $A$ form a base for the topology on $A$, we can find a $B \in \operatorname{Cyl} A$ with $C \subset B$ and $C^{\prime} \cap B=\varnothing$. Since $B \in M_{\lambda}$, we get $\lambda\left(C \cup C^{\prime}\right)=\lambda(C)+\lambda\left(C^{\prime}\right)$. The $\sigma$ additivity of $\lambda$ on $K_{\Omega}$ then follows from the fact that, for any ascending sequence $\alpha, \lambda\left(\bigcup_{n \in \omega} \alpha_{n}\right)=\lim _{n} \lambda\left(\alpha_{n}\right)$ (see Sion [11] Th. 3.3]).

(2) Given $C \in \mathscr{C}$ and neighborhood $U$ of $o$, from the definition of $\lambda$, there exists a sequence $B$ in Cyl $A$ which covers $C$ and such that

$$
C \subset A \subset \bigcup_{n \in \omega} B_{n} \Longrightarrow \lambda(A)-\lambda(C) \in U .
$$

Let $V$ be a sequence of neighborhoods of $o$ with

$$
\sum_{n=0}^{k} V_{n} \subset U
$$

for $k \in \omega$,

$B_{n}=p_{E_{n}}^{-1}\left[\beta_{n}\right]$ with $E_{n} \in \mathscr{F}$ and $\beta_{n} \in M_{E_{n}}$.

Since $\mu$ is a Radon system, for each $n \in \omega$, there exists an open $\gamma_{n} \subset S_{E_{n}}$ such that $\beta_{n} \subset \gamma_{n}$ and $\left(\gamma_{n} \sim \beta_{n}\right)$ is $V_{n}$-small. Since $C \in \mathscr{C}$ there exists $k \in \omega$ such that if

$$
G=\bigcup_{n=0}^{k} p_{E_{n}}^{-1}\left[\gamma_{n}\right]
$$

then $C \subset G$. Moreover $G$ is open, $G \in \operatorname{Cyl} \Lambda$ and

$$
C \subset A \subset G \Longrightarrow \lambda(A)-\lambda(C) \in 2 U \text {. }
$$

Note that if $C \subset \Omega$ then $C \subset(G \cap \Omega),(G \cap \Omega)$ is open in $\Omega$, and $(G \cap \Omega) \in \operatorname{Cyl} \Omega$.

(3) Choose any $E \in \mathscr{F}$ with $E_{n}<E$ for $n=0, \cdots, k$ and let

$$
\gamma=\bigcup_{n=0}^{k} r_{E_{n} E}^{-1}\left[\gamma_{n}\right] \text {. }
$$

Then $G=p_{E}^{-1}[\gamma]$ and, for any $F \in \mathscr{F}$ with $E<F$ and any $\alpha \in M_{F}$,

$$
\begin{aligned}
p_{F}[C] \subset \alpha \subset r_{E F}^{-1}[\gamma] & \Longrightarrow C \subset p_{F}^{-1}[\alpha] \subset p_{E}^{-1}[\gamma]=G \\
& \Longrightarrow \lambda\left(p_{F}^{-1}[\alpha]\right)-\lambda(C) \in 2 U \\
& \Longrightarrow \mu_{F}(\alpha)-\lambda(C) \in 2 U .
\end{aligned}
$$

and therefore

$$
\mu_{F}\left(p_{F}[C]\right)-\lambda(C) \in 3 U
$$

REMARks. When $\tau_{A}$ is $\sigma$-additive and $\lambda=\tau_{A}^{*}$, (1) in view of Lemma 2.4 and theorem 0.6 , we see that $\left(\lambda / \mathscr{K}_{2}\right)$ generates a Radon 
outer measure $\rho$ on $\Omega$.

(2) Lemma 2.4.3 gives a characterization of $\left(\lambda / \mathscr{K}_{\Omega}\right)$ directly in terms of $\mu$, which poins out that, when $\Gamma=\boldsymbol{R}$, the processes for constructing a Radon limit measure followed respectively by Mallory and Sion in [4] and by C. Scheffer in [9] are essentially the same.

Putting all the pieces together, we get the following.

THEOREM 2.5. $\mu$ has a Radon limit on $\Omega$ iff $\mu$ is $\mathscr{K}_{\Omega}$-tight. ${ }^{(3)}$

Proof. (1) Suppose $\mu$ has a Radon limit $\rho$ on $\Omega$. Then, for any neighborhood $U$ of $o$, there exists $C \in \mathscr{K}_{2}$ such that

$$
\alpha \subset \Omega \sim C \Longrightarrow \rho(\alpha) \in U \text {. }
$$

For any $E \in \mathscr{F}$, since

$$
\Omega \cap p_{E}^{-1}\left[S_{E} \sim p_{E}[C]\right] \subset \Omega \sim C,
$$

we see that $\left(S_{E} \sim p_{E}[C]\right)$ is $U$-small. Thus, $\mu$ is $\mathscr{K}_{\Omega}$-tight.

(2) Suppose $\mu$ is $\mathscr{K}_{\Omega}$-tight. Then, by Theorem 1.11, $\Omega$ and hence a fortiori $\Lambda$ is almost sequentially maximal so, by Theorem 2.2, $\tau_{A}$ is $\sigma$-additive. Let $\lambda=\tau_{A}^{*}$ and, using Lemma 2.4 and Theorem 0.6, let $\rho$ be the Radon outer measure on $\Omega$ generated by $\left(\lambda / \mathscr{K}_{\Omega}\right)$. We shall check that $\rho$ is a limit of $\mu$ on $\Omega$. Let $E \in \mathscr{F}, A \in M_{E}$ and $\alpha=\Omega \cap p_{\bar{E}}^{-1}[A]$.

(2a) To see that $\alpha \in M_{\rho}$, given any neighborhood $U$ of $o$, choose a compact $A^{\prime}$ and open $A^{\prime \prime}$ such that $A^{\prime} \subset A \subset A^{\prime \prime}$ and $\left(A^{\prime \prime} \sim A^{\prime}\right)$ is $U$-small and let $\alpha^{\prime}=\Omega \cap p_{E}^{-1}\left[A^{\prime}\right]$ and $\alpha^{\prime \prime}=p_{E}^{-1}\left[A^{\prime \prime}\right]$. Then $\alpha^{\prime}, \alpha^{\prime \prime} \in M_{\rho}$, $\alpha^{\prime} \subset \alpha \subset \alpha^{\prime \prime}$ and, for any $C \in \mathscr{K}_{\Omega}$, by Lemma 2.4.3, $C \subset \alpha^{\prime \prime} \sim \alpha^{\prime} \Rightarrow \rho(C)=$ $\lambda(C) \in U$. Hence, for any $\beta \subset \alpha^{\prime \prime} \sim \alpha^{\prime}$, we have $\rho(\beta) \in 2 U$. Thus, by Lemma 0.7, $\alpha \in M_{\rho}$.

(2b) To see that $\rho(\alpha)=\mu_{E}(A)$, given any neighborhood $U$ of 0 , choose $C_{1}, C_{2} \in \mathscr{K}_{Q}$ and $K \in \mathscr{K}_{E}$ such that

(i) $\left(S_{F} \sim p_{F}\left[C_{1}\right]\right)$ is $U$-small for all $F \in \mathscr{F}$,

(ii) $C_{2} \subset \alpha$ and $\beta \subset \alpha \sim C_{2} \Rightarrow \rho(\beta) \in U$,

(iii) $K \subset A$ and $(A \sim K)$ is $U$-small,

and let

$$
C=\left(C_{1} \cap p_{E}^{-1}[K]\right) \cup C_{2} \text {. }
$$

Then $C \in \mathscr{K}_{\Omega}, C_{2} \subset C \subset \alpha$, so $\rho(\alpha)-\rho(C) \in U$ and, for any $F \in \mathscr{F}$ with $E<F$,

3 The case of real-valued measures is treated in the forthcoming "Radón measures on topological spaces," (Tata Institute, Bombay) by Laurent Schwartz, Thms. I. 20, I. 21. 


$$
\begin{aligned}
& r_{E F}^{-1}[K] \sim p_{F}[C] \subset S_{F} \sim p_{F}\left[C_{1}\right] \\
& p_{F}[C] \sim r_{E F}^{-1}[K] \subset r_{E F}^{-1}[A \sim K]
\end{aligned}
$$

so

$$
\mu_{F}\left(p_{F}[C]\right)-\mu_{F}\left(r_{E F}^{-1}[K]\right) \in 2 U
$$

and

$$
\mu_{F}\left(p_{F}[C]\right)-\mu_{E}(A) \in 3 U \text {. }
$$

By Lemma 2.4.3 therefore, $\rho(C)-\mu_{E}(A) \in 4 U$ and so

$$
\rho(\alpha)-\mu_{E}(A) \in 5 U \text {. }
$$

REMARK. For $\Gamma=R$, the above theorem was first given by Mourier [7] and later extended by Prohorov [8]. Minlos [6] attributes a similar theorem to V. Erohin.

3. Cylinder measures. We shall now apply the ideas of the previous sections to the study of cylinder measures.

Given any vector spaces $X, Y$ over a field $\Phi$, let $\mathscr{F}$ be the family of all finite dimensional subspaces of $X$ directed by $\subset$;

$S_{E}$ be the set of all linear functions on $E$ to $Y$ for $E \in \mathscr{F}$;

$$
r_{E F}: f \in S_{F} \longrightarrow(f / E) \in S_{E} \text { for } E, F \in \mathscr{F} \text { with } E \subset F .
$$

Definitions 3.1. (1) $\mu$ is a cylinder measure over $(X, Y)$ iff $\mu$ is a monotone, inverse system of measures over $(S, r)$.

(2) When the $S_{E}$ are topological spaces for $E \in \mathscr{F}, \mu$ is a Radon cylinder measure over $(X, Y)$ iff $\mu$ is a Radon system of measures over $(S, r)$.

When $\Phi=Y=R$, the $S_{E}$ are finite dimensional spaces for $E \in \mathscr{F}$ and hence have a canonical locally convex topology with respect to which the $r_{E F}$ are obviously continuous.

If, in addition, we let $\Gamma=R, \mu$ be a Radon system, $X$ be a topological vector space, $\Omega$ be the topological dual of $X$, and $p_{E}: f \in \Omega \rightarrow$ $(f / E) \in S_{E}$ for $E \in \mathscr{F}$ then, in our terminology, $\tau_{\Omega}$ is the function referred to as a cylinder measure by most workers. Even when the definition of cylinder measure is formulated as an inverse system of measures, the system is given in terms of $\Omega$ (see e.g., Minlos [6], Schwartz [10]).

Thus, besides allowing more general sets for $\Phi, \Gamma$ and $Y$, our definition of cylinder measure is free of any a priori choice of a target space $\Omega$ on which to place a limit measure and permits us therefore to consider a variety of sets $\Omega$. Let $\Lambda$ be the set of all 
linear functions on $X$ to $Y$ and $p_{E}: f \in \Lambda \rightarrow(f \mid E) \in \mathbb{S}_{E}$ for $E \in \mathscr{F}$.

One justification for the choice of $(\Lambda, p)$ a limit for $(S, r)$ is that it can be identified in an obvious way with the canonical inverse limit $(L, \pi)$ where

$$
L=\left\{f \in \prod_{E \in \mathscr{F}} S_{E}: r_{E F}\left(f_{F}\right)=f_{E} \text { for } E, F \in f \text { with } E \subset \mathscr{F}\right\}
$$

and

$$
\pi_{E}(f)=f_{E} \quad \text { for } f \in L \text { and } E \in \mathscr{F} \text {. }
$$

Assumptions 3.2. For the remainder of this section, we assume $S_{E}$ is a Hausdorff, regular topological space for $E \in \mathscr{F} ; r_{E F}$ is continuous for $E, F \in \mathscr{F}$ with $E \subset F ; \mu$ is a Radon cylinder measure over $(X, Y) ; \Omega \subset \Lambda$.

The results of the previous sections then yield.

THEOREM 3.3. $\mu$ has a limit on 4 .

Proof. $\Lambda$ is clearly sequentially maximal so Theorem 2.2 applies.

THEOREM 3.4. If $\Omega$ is almost sequentially maximal then $\mu$ has a limit on $\Omega$.

\section{Proof. Apply Theorem 1.10.}

THEOREM 3.5. If $\Omega$ is a regular topological space, $\left(p_{E} / \Omega\right)$ is continuous for $E \in \mathscr{F}$, and $\mathscr{K}$ is the family of compact subsets of $\Omega$ then

(1) $\mu$ has a Radon limit on $\Omega$ iff $\mu$ is $\mathscr{K}$-tight.

(2) If $\mu$ has a Radon limit on $\Omega$ then $\Omega$ is almost sequentially maximal.

Proof. Apply Theorems 2.5 and 1.11 .

We should point out that Theorem 3.4 would have very limited applicability if we replaced "almost sequential maximality" by "sequential maximality" in view of Theorem 3.5 and the following.

Observation. If $X$ is a topological vector space over $\boldsymbol{R}$ with an infinite bounded linearly independent subset, $Y=R$ and $\Omega$ is the topological dual of $X$ then $\Omega$ is not sequentially maximal.

Proof. Let $\left\{e_{n} ; n \in \omega\right\}$ be a bounded linearly independent subset of $X$, let $X_{0}$ denote its span and $f$ be the linear functional on $X_{0}$ with $f\left(e_{n}\right)=n$ for $n \in \omega$. If $\Omega$ were sequentially maximal, there 
would exist $g \in \Omega$ with $g / X_{0}=f$, which is impossible since $f$ is not continuous on $X_{0}$.

\section{REFERENCES}

1. A. Badrikian, Ramarques sur les theoremes de Bochner et P. Levy, Symp. on Prob. Methods in Anal.; Lect. Notes (31). Springer (1967).

2. S. Bochner, Harmonic Analysis and the Theory of Probability, Univ. of California Press, Berkeley, Calif. (1955).

3. J. Choksi, Inverse limits of measure spaces, Proc. London Math. Soc., 8 (1958), $321-342$.

4. D. Mallory and M. Sion, Limits of inverse systems of measures, Ann. Inst. Fourier, 21 (1971), 25-57.

5. M. Metivier, Limites projectives de mesures, Martingales applications, Ann. di Mathematica, 63 (1963), 225-352.

6. R. Minlos, Generalized random processes, Inst. of Math. Stats., Selected Transl. in Math. Stats. and Prob., 3 (1962), 291-313.

7. E. Mourier, Elements aleatoires dans un espace de Banach, Ann. Inst. Henri Poincare, 13 (1953), 261-244.

8. Yu. Prohorov, The methods of characteristic functionals, Proc. 4 th. Berk. Symp. Math. Stats. and Prob., 2 (1960), 403-419.

9. C. Scheffer, Sur l'existence de la limite projective ... C. R. Acad. Sci., Paris, 269 A, No. 4 (28 July 1969), 205-207.

10. L. Schwartz, Extension du theoreme de Sazonov-Minlos, C. R. Acad. Sci., 266 A (3. Jan. 1968), 7-9.

11. M. Sion, Outer measures with values in a topological group, Proc. London Math. Soc., 19 (1969), 89-106.

12. Lectures on Vector-Valued Measures, Univ. of Br. Col. (1969-70).

Received March 9, 1971.

The University of British Columbia 


\section{PACIFIC JOURNAL OF MATHEMATICS}

\section{EDITORS}

\section{H. SAMELSON}

Stanford University

Stanford, California 94305

C. R. Новву

University of Washington Seattle, Washington 98105

\section{J. DuGundJI}

Department of Mathematics University of Southern California Los Angeles, California 90007

RICHARD ARENS

University of California Los Angeles, California 90024

\section{ASSOCIATE EDITORS}
E. F. BECKENBACH
B. H. NeumanN
F. WOLF
K. YoSHIDA

\section{SUPPORTING INSTITUTIONS}

\author{
UNIVERSITY OF BRITISH COLUMBIA \\ CALIFORNIA INSTITUTE OF TECHNOLOGY \\ UNIVERSITY OF CALIFORNIA \\ MONTANA STATE UNIVERSITY \\ UNIVERSITY OF NEVADA \\ NEW MEXICO STATE UNIVERSITY \\ OREGON STATE UNIVERSITY \\ UNIVERSITY OF OREGON \\ OSAKA UNIVERSITY
}

\author{
UNIVERSITY OF SOUTHERN CALIFORNIA \\ STANFORD UNIVERSITY \\ UNIVERSITY OF TOKYO \\ UNIVERSITY OF UTAH \\ WASHINGTON STATE UNIVERSITY \\ UNIVERSITY OF WASHINGTON \\ $*{ }^{*}$
AMERICAN MATHEMATICAL SOCIETY
NAVAL WEAPONS CENTER
}

The Supporting Institutions listed above contribute to the cost of publication of this Journal, but they are not owners or publishers and have no responsibility for its content or policies.

Mathematical papers intended for publication in the Pacific Journal of Mathematics should be in typed form or offset-reproduced, (not dittoed), double spaced with large margins. Underline Greek letters in red, German in green, and script in blue. The first paragraph or two must be capable of being used separately as a synopsis of the entire paper. The editorial "we" must not be used in the synopsis, and items of the bibliography should not be cited there unless absolutely necessary, in which case they must be identified by author and Journal, rather than by item number. Manuscripts, in duplicate if possible, may be sent to any one of the four editors. Please classify according to the scheme of Math. Rev. Index to Vol, 39. All other communications to the editors should be addressed to the managing editor, Richard Arens, University of California, Los Angeles, California, 90024.

50 reprints are provided free for each article; additional copies may be obtained at cost in multiples of 50 .

The Pacific Journal of Mathematics is issued monthly as of January 1966. Regular subscription rate: $\$ 48.00$ a year (6 Vols., 12 issues). Special rate: $\$ 24.00$ a year to individual members of supporting institutions.

Subscriptions, orders for back numbers, and changes of address should be sent to Pacific Journal of Mathematics, 103 Highland Boulevard, Berkeley, California, 94708.

PUBLISHED BY PACIFIC JOURNAL OF MATHEMATICS, A NON-PROFIT CORPORATION

Printed at Kokusai Bunken Insatsusha (International Academic Printing Co., Ltd.), 270, 3-chome Totsuka-cho, Shinjuku-ku, Tokyo 160, Japan. 


\section{Pacific Journal of Mathematics}

\section{Vol. 44, No. $2 \quad$ June, 1973}

Tsuyoshi Andô, Closed range theorems for convex sets and linear liftings . . . . . . 393

Richard David Bourgin, Conically bounded sets in Banach spaces . . . . . . . . . 411

Robert Jay Buck, Hausdorff dimensions for compact sets in $R^{n} \ldots \ldots \ldots \ldots \ldots \ldots . \ldots 421$

Henry Cheng, A constructive Riemann mapping theorem ................ 435

David Fleming Dawson, Summability of subsequences and stretchings of

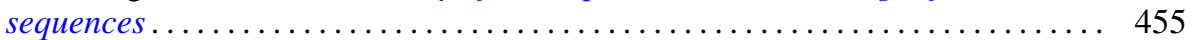

William Thomas Eaton, A two sided approximation theorem for 2-spheres ....... 461

Jay Paul Fillmore and John Herman Scheuneman, Fundamental groups of compact complete locally affine complex surfaces ....................... 487

Avner Friedman, Bounded entire solutions of elliptic equations . . . . . . . . . . . 497

Ronald Francis Gariepy, Multiplicity and the area of an $(n-1)$ continuous

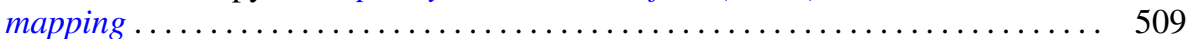

Andrew M. W. Glass, Archimedean extensions of directed interpolation groups . . . . 515

Morisuke Hasumi, Extreme points and unicity of extremum problems in $H^{1}$ on

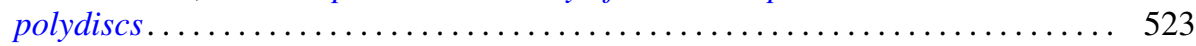

Trevor Ongley Hawkes, On the Fitting length of a soluble linear group . . . . . . 537

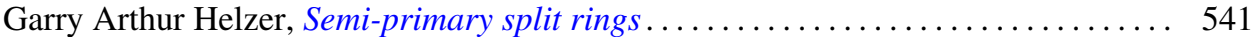

Melvin Hochster, Expanded radical ideals and semiregular ideals . . . . . . . . . 553

Keizō Kikuchi, Starlike and convex mappings in several complex variables . . . . . . 569

Charles Philip Lanski, On the relationship of a ring and the subring generated by its

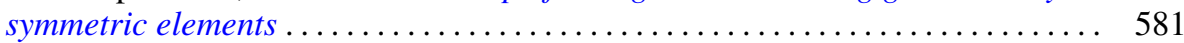

Jimmie Don Lawson, Intrinsic topologies in topological lattices and semilattices ........................................... 593

Roy Bruce Levow, Counterexamples to conjectures of Ryser and de Oliveira ...... 603

Arthur Larry Lieberman, Some representations of the automorphism group of an infinite continuous homogeneous measure algebra ..........

William George McArthur, $G_{\delta}$-diagonals and metrization theorems $\ldots .$.

James Murdoch McPherson, Wild arcs in three-space. II. An invariant of

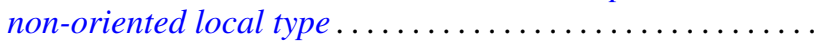

H. Millington and Maurice Sion, Inverse systems of group-valued measures ...

C. Edward Moore, Concrete semispaces and lexicographic separation of convex

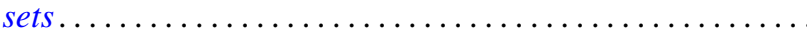

Jingyal Pak, Actions of torus $T^{n}$ on $(n+1)$-manifolds $M^{n+1}$.

Merrell Lee Patrick, Extensions of inequalities of the Laguerre and Turán type . . . . 675

Harold L. Peterson, Jr., Discontinuous characters and subgroups of finite index. . . . 683

S. P. Philipp, Abel summability of conjugate integrals . . . . . . . . . . . . . 693

R. B. Quintana and Charles R. B. Wright, On groups of exponent four satisfying an

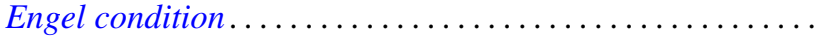

Marlon C. Rayburn, On Hausdorff compactifications. . . . . . . . . .

Martin G. Ribe, Necessary convexity conditions for the Hahn-Banach theorem in

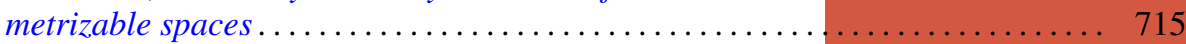

Ryōtarō Satō, On decomposition of transformations in infinite measure spaces .... 733

Peter Drummond Taylor, Subgradients of a convex function obtained from a

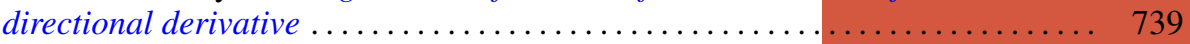

James William Thomas, A bifurcation theorem for $k$-set contractions . . . . . . . . 749 Clifford Edward Weil, A topological lemma and applications to real functions . . . . 757

Stephen Andrew Williams, A nonlinear elliptic boundary value problem . . ....... 767

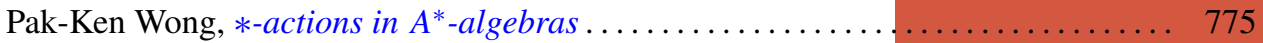

